\title{
BMJ
}

\section{Diagnosis-specific sickness absence as a predictor of mortality: the Whitehall II prospective cohort study}

\author{
Jenny Head, reader in medical and social statistics, Jane E Ferrie, senior research fellow, ${ }^{1}$ Kristina \\ Alexanderson, professor, ${ }^{2}$ Hugo Westerlund, senior researcher, ${ }^{3}$ Jussi Vahtera, research professor, ${ }^{4}$ Mika \\ Kivimäki, professor ${ }^{1,4}$
}

${ }^{1}$ Department of Epidemiology and Public Health, University College London, London

${ }^{2}$ Department of Clinical Neuroscience, Section of Personal Injury Prevention, Karolinska Institutet, Berzelius väg 3, 17177 Stockholm, Sweden

${ }^{3}$ Stress Research Institute, Stockholm University, SE-106 91, Stockholm

${ }^{4}$ Finnish Institute of Occupational Health, Topeliuksenkatu 41 aA, FIN-00250 Helsinki, Finland Correspondence to: J Head j.head@ucl.ac.uk

BMJ 2008;:1-7 doi:10.1136/bmj.a1469

\section{ABSTRACT}

Objective To investigate whether knowing the diagnosis for sickness absence improves prediction of mortality. Design Prospective cohort study established in 1985-8. Sickness absence records including diagnoses were obtained from computerised registers.

Setting 20 civil service departments in London. Participants 6478 civil servants aged 35-55 years. Main outcome measures All cause, cardiovascular, and cancer mortality until 2004, average follow-up 13 years. Results After adjustment for age, sex, and employment grade, employees who had one or more medically certified spells of sickness absence ( $>7$ days) in a three year period had a mortality $1.7(95 \% \mathrm{Cl} 1.3$ to 2.1$)$ times greater than those with no medically certified spells. Inclusion of diagnoses improved the prediction of all cause mortality $(P=0.03)$. The hazard ratio for mortality was 4.7 (2.6 to 8.5) for absences with circulatory disease diagnoses, 2.2 (1.4 to 3.3) for surgical operations, and 1.9 (1.2 to 3.1) for psychiatric diagnoses. Psychiatric absences were also predictive of cancer mortality (2.5 (1.3 to 4.7)). Associations of infectious, respiratory, and injury absences with overall mortality were less marked (hazard ratios from 1.5 to 1.7 ), and there was no association between musculoskeletal absences and mortality. Conclusions Major diagnoses for medically certified absences were associated with increased mortality, with the exception of musculoskeletal disease. Data on sickness absence diagnoses may provide useful information to identify groups with increased health risk and a need for targeted interventions.

\section{INTRODUCTION}

The annual rate of medically certified spells of sickness absence seems to be a good global measure of health. ${ }^{12}$ It reflects day to day functioning in occupational settings and predicts mortality at least as well as more established indicators of health such as poor self rated health, minor psychiatric morbidity, and presence of longstanding physical illness, disability, or infirmity. ${ }^{3}$ The reasons for such good predictive power of the overall rate of sickness absence are not well understood. According to one hypothesis, sickness absence spells may be a strong predictor of mortality because they capture a wide range of illnesses that employees experience. ${ }^{3}$ An alternative view is that the increased risk of early mortality is confined to specific reasons for absence such as cancer, heart disease, or surgery for which increased mortality would be expected.

One way to clarify the reasons for the predictive value of sickness absence in relation to mortality is to include diagnoses for sickness absence in the analysis. If sickness absence predicts mortality because it captures the full range of illnesses which separately or in combination increase death risk, then specific diagnoses for absences would not improve prediction of mortality compared with the overall sickness absence rate. In contrast, sickness absence diagnoses would contribute to predictive power if specific diagnoses underlie the association between overall sickness absence and mortality or if the strength of association differs according to diagnosis.

In this study, we investigated whether diagnosisspecific sickness absence predicts subsequent mortality from all causes and from specific causes in the Whitehall II cohort of British civil servants. We also determined whether including information on diagnoses for sickness absence improved the prediction of mortality compared with overall sickness absence rates irrespective of diagnoses.

\section{METHODS}

\section{Participants}

The target population for the Whitehall II study was all London based office staff aged 35-55 working in 20 civil service departments. Baseline screening was carried out between 1985 and $1988 .{ }^{4}$ With a response rate of $73 \%$, the final cohort consisted of 10308 (6895 men, 3413 women). The true response rate was higher, however, because about $4 \%$ of those invited were not eligible for inclusion. Ethical approval for the Whitehall II study was obtained from the University College London Medical School committee on the ethics of human research.

\section{Sickness absence}

We obtained computerised sickness absence records from 1 January 1985 onwards from civil service pay 
centres for 9179 employees. These records included the start and end dates of all absences. For absences longer than seven calendar days, a medical certificate was required.

Diagnosis for absence was recorded in 12 of the 20 participating departments (61\% of participants) from 1985 onwards and was recorded for all 20 departments from 1991 onwards. The exposure in this study was medically certified sickness absence during the first three years for which diagnosis was recorded. For the 12 departments with full records of diagnosis for absence, we used data on sickness absence during the three years after the baseline screening date as the exposure; for the other eight departments, where recording of diagnosis was only available from 1991 onwards, we used data on sickness absence during the three years from 1 January 1991 as the exposure time.

Sickness absence diagnoses were coded by the civil service using a detailed coding system based on the international classification of diseases (ICD-8). The coding system was modified in 1990, with codes in the old system (942 codes) mapped on to a smaller number of codes (586) in the new system. We converted the civil service codes to disease categories using the morbidity coding system of the Royal College of General Practitioners (RCGP). The RCGP disease categories are similar to the ICD-8 chapter headings. We

Modified RCGP disease categories used in this study

Code 1-Infectious and parasitic diseases
Code 2-Neoplasms
Code 3-Endocrine, nutritional, and metabolic diseases
Code 4-Diseases of blood and blood forming organs
Code 5-Psychoses
Code 6-Diseases of system
Code 7-Diseases of eye
Code 8 -Diseases of ear
Code 9-Cardiovascular diseases
Code 10-Cerebrovascular diseases
Code 11-Peripheral vascular diseases
Code 12-Diseases of respiratory system
Code 13-Diseases of digestive system
Code 14-Diseases of genitourinary system
Code 15-Complications of pregnancy, childbirth, and puerperium
Code 16-Diseases of skin and subcutaneous tissue
Code 17-Diseases of musculoskeletal system and connective tissue
Code 20-Symptoms, signs, and ill-defined conditions
Code 23-Injury and poisoning
Code 35-General medical examinations and screening
Code 37-Surgical operations
Code 38-Gastrointestinal
Code 39-Headache and migraine
Code 40-Neurosis
Code 41-Neurosis ill-defined
Cons

modified the RCGP classification by adding four extra disease categories (gastrointestinal, headache and migraine, neurosis, neurosis ill-defined) that accounted for a high proportion of sickness absence spells. Further details on the coding of diagnoses into the 25 disease categories (see box) have been reported previously. $^{5}$

We assessed the validity of the civil service diagnosis codes by obtaining information from participants' general practitioners for all absence spells lasting $>21$ days in 1985-90: the civil service codes and general practitioners showed agreement on disease category for $64 \%$ of these absences. ${ }^{6} \mathrm{We}$ also checked the sickness absence records for inconsistencies: we merged overlapping, consecutive, or duplicate spells of sickness absence after taking account of weekends and public holidays, and used the final diagnosis for absence when we merged contiguous spells.

\section{Mortality}

We obtained mortality data for almost all the participants $(n=10297)$ up to 30 September 2004 from the National Health Service Central Register mortality register. In addition to deaths from all causes, we analysed deaths from cardiovascular diseases (ICD9 codes 390-459, ICD-10 codes I00-I99) and cancer (ICD-9 140-208, ICD-10 C00-C97). Smoking related cancers were considered to be ICD-9 codes 140, 141, 143-150, 157, 160-163, 188, and 189 and ICD-10 codes C00-C06, C09-C15, C25, C30-C34, C38, and C64C68. ${ }^{7}$

\section{Other measures}

Smoking, alcohol consumption (units consumed in previous 7 days), presence of hypertension (use of antihypertensive drugs or blood pressure $>160 / 95 \mathrm{~mm} \mathrm{Hg})$, body mass index (weight $(\mathrm{kg}) /\left(\right.$ height $\left.(\mathrm{m})^{2}\right)$, self rated health over the past 12 months, and presence of longstanding illness, disability, or infirmity were recorded at baseline. We created a composite physical illness indicator (diabetes, diagnosed heart trouble, electrocardiographic abnormalities, hypertension, and respiratory illness). Self reported diagnosis of cancer was recorded at baseline and at phase 4 follow-up of the Whitehall II study (1995-6).There were missing data for the measure of longstanding illness as this was introduced only after the start of the baseline survey: where data were missing, we used reported longstanding illness from the phase 2 follow-up survey (1989-90).

\section{Statistical analysis}

For each employee, we computed the numbers of medically certified spells ( $>7$ days) of sickness absence for each diagnostic category during the three year exposure period. Diagnostic categories with small numbers of participants were combined into an "other" category. Follow-up for mortality was from the first day after the three year exposure period until 30 September 2004. We used Cox's proportional 
Civil service staff screened at baseline in 1985-8 ( $\mathrm{n}=10$ 308)

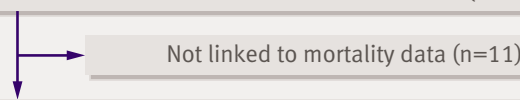

Linked to mortality data $(n=10297)$

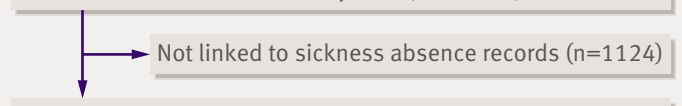

Linked to sickness absence records $(n=9173)$

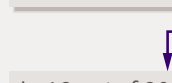

In 12 out of 20 departments, diagnoses for sickness

absences recorded from

baseline screening date

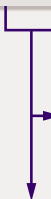

Staff with incomplete data for three

year absence exposure period $(n=2695)$ :

Died $(n=120)$

Left civil service $(n=2575)$

Staff with complete three year record of sickness absences including diagnosis $(n=6478)$

$\downarrow$

Follow up for mortality to 30 September 2004 (288 deaths)

Flow of participants through study

hazard models to estimate hazard ratios for having taken one or more spells of absence for each sickness absence diagnostic category. The reference group was participants who had no sickness absence spells lasting $>7$ days during the exposure period. All analyses were adjusted for sex, age (in 5 year groups), and employment grade. If participants had two (or more) spells of sickness absence in different diagnostic categories, they were included in both categories.

Using the likelihood ratio $\chi^{2}$ test, we tested for an improvement in predictive power for mortality by taking account of absence diagnoses. We compared a multivariate model including indicators for the main diagnostic categories with the simpler model ignoring diagnoses (any medically certified sickness absences versus no such absences). We tested for the possibility of differences in associations for women and men by including an interaction term with sex. Further analyses adjusted for other known predictors of mortality (smoking, alcohol consumption, hypertension, and body mass index). We tested the proportional hazards assumption by including an interaction of each diagnosis-specific absence variable with the $\log$ of follow-up time; in all cases, this interaction was not statistically significant. We used the SAS 9.1 program for all analyses.

\section{RESULTS}

Excluded participants

Of the 10308 participants in the Whitehall II study, 3830 were excluded from our analysis (figure). Reasons for exclusion were missing data on mortality $(n=11)$ or absence records $(n=1124)$ and an incomplete three year exposure period $(\mathrm{n}=2695 ; 120$ died and 2575 left the civil service during the exposure period). We compared mortality after 1993 in the remaining sample of 6478 participants with the sample excluded from analysis. After adjustment for age, sex, and employment grade, mortality was lower in the analysed sample than in the excluded sample (hazard ratio $0.81,95 \%$ confidence interval 0.67 to 0.98 ).

\section{Overall sickness absence and mortality}

Of the 6478 participants, 288 died during the follow-up period from the first day after the sickness absence exposure to September 2004. Mean follow-up was 13.2 (SD 2.3, range 0.1-16.1) years. Mortality in the 12 civil service departments where absence diagnoses were recorded from baseline was similar to that in the other eight departments where recording began in 1991 (hazard ratio $0.98,0.74$ to 1.31 ).

Table 1 shows the numbers of participants who had one or more spells of medically certified sickness absence during the three year exposure period. A total of 1906 employees (29\%) had one or more such spells, $18 \%$ having one spell only and $11 \%$ having two or more. Altogether, there were 3214 such spells of absence, with a median length of 16 days.

Table 2 shows the associations of these spells of absence with subsequent mortality, (adjusted for age,

Table 1| Distribution of spells of medically certified sickness absence during three year exposure period among civil service employees

\begin{tabular}{|ccc} 
& $\begin{array}{c}\text { No (\%) of } \\
\text { participants } \\
(n=6478)\end{array}$ & $\begin{array}{c}\text { No (\%) of spells } \\
\text { of sickness } \\
\text { absence }\end{array}$ \\
Any medically certified spells of & $1906(29.4)$ & $3214(100)$
\end{tabular}

sickness absence (lasting $>7$ days)

No of medically certified spells in

three years:

\begin{tabular}{ccc}
\hline 0 & $4572(70.6)$ & - \\
\hline 1 & $1170(18.1)$ & - \\
\hline 2 & $415(6.4)$ & - \\
\hline 3 & $183(2.8)$ & - \\
\hline$>3$ & $138(2.1)$ & - \\
\hline
\end{tabular}

Diagnostic category for medically

certified spells (category code)*

\begin{tabular}{lcc}
\hline Infectious (1) & $244(3.8)$ & $274(8.5)$ \\
\hline Respiratory (12) & $685(10.6)$ & $850(26.4)$ \\
\hline Musculoskeletal (17) & $257(4.0)$ & $305(9.5)$ \\
\hline Circulatory $(9,10,11)$ & $61(0.9)$ & $71(2.2)$ \\
\hline Injury (23) & $230(3.6)$ & $255(7.9)$ \\
\hline Psychiatric $(40,41)$ & $235(3.6)$ & $303(9.4)$ \\
\hline Surgery (37) & $318(4.9)$ & $364(11.3)$ \\
\hline Other (2-8,13-16, 20, 35, 38, 39) & $377(5.8)$ & $461(14.3)$ \\
\hline Not codeable or missing & $289(4.5)$ & $331(10.3)$ \\
\hline Multiple medically certified spellst: & & \\
\hline 2 spells same diagnosis & $105(1.7)$ & - \\
\hline 2 spells different diagnoses & $231(3.7)$ & - \\
\hline 22 spells same diagnosis & $27(0.4)$ & - \\
\hline 22 spells different diagnoses & $214(3.5)$ & - \\
\hline
\end{tabular}

*Modified RCGP morbidity coding system (see text for details). †Among the 6189 participants with no missing or uncodeable diagnoses. 
sex, and employment grade). Among the participants who had had one or more medically certified absence in the three year exposure period mortality was 1.7 times higher than among those with no such absences, and further adjustment for self rated health, longstanding illness, and the composite physical illness indicator had little effect on this (hazard ratio 1.59, 1.16 to 2.17). We also found a dose-response association between rates of medically certified absence with subsequent mortality: compared with having no medically certified absences, the hazard ratio for having two or more medically certified absences was 1.97 (1.43 to 2.71) and for having one such absence was 1.48 (1.11 to 1.98$)$.

\section{Diagnosis-specific sickness absence and mortality}

The commonest diagnostic categories were respiratory, surgery, musculoskeletal, psychiatric, infectious diseases, and injury - which together accounted for $73 \%$ of all medically certified absences in the three year exposure period. Among those participants who had two or more absences, $77 \%$ had absences attributed to two or more of the 25 different diagnostic categories.

As shown in table 2, medically certified absences attributed to infectious diseases, respiratory diseases, and injury had similar hazard ratios for mortality, ranging from 1.5 to 1.7 . The increased mortality risk was greater, but not significantly so, for spells of absence with a non-psychotic psychiatric diagnosis (hazard ratio 1.9), and this increased risk was seen with both subcategories "neurosis" (1.9) and "neurosis illdefined" (2.0). Hazard ratios were higher for sickness absences attributed to circulatory disease (4.7) and surgical operations (2.2). Fewer than 10 people had sickness absence attributed to cancer diagnoses; this group had a particularly high hazard ratio of $21.3(6.7$ to 67.4). Those having absence with a musculoskeletal diagnosis did not have increased mortality compared

Table 2 | Hazard ratios for all cause mortality (adjusted for age, sex, and employment grade) among 6478 civil service employees by specific diagnoses for medically certified spells of sickness absence

\begin{tabular}{|c|c|c|}
\hline Diagnostic category (category code)* & No of participants (deaths) & Hazard ratio $(95 \% \mathrm{Cl})$ \\
\hline No medically certified sickness absence & $4570(161)$ & 1.00 \\
\hline Any medically certified absence (all diagnoses): & $1906(127)$ & $1.66(1.30$ to 2.13$)$ \\
\hline Infectious and parasitic diseases (1) & $244(15)$ & 1.51 (0.88 to 2.59$)$ \\
\hline Circulatory (9-11) & $61(12)$ & $4.68(2.58$ to 8.51$)$ \\
\hline Diseases of respiratory system (12) & $685(46)$ & 1.63 (1.15 to 2.29$)$ \\
\hline $\begin{array}{l}\text { Diseases of musculoskeletal system and } \\
\text { connective tissue (17) }\end{array}$ & $257(12)$ & 1.04 (0.57 to 1.89$)$ \\
\hline Injury and poisoning (23) & $230(17)$ & $1.66(0.99$ to 2.78$)$ \\
\hline Surgical operations (37) & $318(27)$ & $2.16(1.42$ to 3.26$)$ \\
\hline Psychiatric $(40,41)$ : & $235(19)$ & $1.91(1.17$ to 3.11$)$ \\
\hline Neurosis (40) & $87(7)$ & $1.91(0.89$ to 4.11$)$ \\
\hline Neurosis ill-defined (41) & $164(14)$ & 2.03 (1.16 to 3.55$)$ \\
\hline Other $(2-8,13-16,20,35,38,39)$ & $377(27)$ & 1.78 (1.17 to 2.70$)$ \\
\hline Diagnosis not codeable or missing & $289(22)$ & 2.09 (1.33 to 3.30) \\
\hline
\end{tabular}

with participants who took no medically certified absences (hazard ratio 1.0).

Comparison of our models with and without sickness absence diagnoses indicated that inclusion of diagnoses significantly improved the prediction of mortality $(\mathrm{P}$ for improvement in $\left.\chi^{2}=0.03\right)$. This statistical test of improvement in model fit is equivalent to testing whether hazard ratios for mortality vary by diagnosis. The only diagnosis where the hazard ratio for all cause mortality significantly exceeded the hazard ratio of 1.66 for all absences was circulatory disease $(\mathrm{P}<0.001)$.

None of the interactions between diagnostic category and sex was significant. Having multiple absences with two or more different diagnoses during the three year exposure period was associated with slightly higher mortality, but this was not significant (hazard ratio $1.34,0.75$ to 2.39 , for $\geq 2$ absences with different diagnoses $v \geq 2$ absences with same diagnosis).

We repeated analyses after excluding the 31 employees who died in the three years immediately after the three year sickness absence exposure period, to exclude the possibility that absence close to death might account for our findings. The hazard ratio for any medically certified absence compared with no such absence was 1.59 (1.23 to 2.07). Likewise, hazard ratios for diagnosis-specific absence were also hardly altered (results not shown).

\section{Sickness absence and cause-specific mortality}

Table 3 presents associations between medically certified spells of absence and cause-specific mortality. The two leading causes of death were cancer (144 deaths) and cardiovascular mortality (72 deaths), accounting for $50 \%$ and $25 \%$ of all deaths. Other causes (68 deaths) accounted for $24 \%$ of all deaths. Cause was unknown for four deaths. Among the cancer deaths, 30\% were assessed as smoking related.

Medically certified spells of sickness absence were associated with both cardiovascular mortality (hazard ratio 2.0) and cancer mortality (hazard ratio 1.7). Absence attributable to psychiatric disorder showed a stronger association with cancer mortality (hazard ratio 2.5) than with cardiovascular mortality (hazard ratio 1.2), but the difference was not significant. The association with cancer mortality was seen for both psychiatric categories - "neurosis" (hazard ratio 2.2, 0.8 to 6.2$)$ and "neurosis ill-defined" (3.0, 1.5 to 5.9) and was also observed in the subgroup whose absences were all attributable to psychiatric disorder (hazard ratio 2.4). To exclude the possibility that this association might be explained by higher rates of sickness absence for psychiatric causes among people with cancer diagnosed, we repeated the analysis excluding the 166 participants with a self reported diagnosis of cancer either at baseline or follow-up in 1995-6. In the subgroup without cancer, participants who had absences with a psychiatric diagnosis had cancer mortality 2.4 times higher $(95 \%$ CI 1.2 to 4.6$)$ than did those with no absence.

Otherwise, rates of diagnosis-specific absence tended to show stronger associations with 
Table 3 | Hazard ratios for cause-specific mortality (adjusted for age, sex, and employment grade) among 6478 civil service employees by specific diagnoses for medically certified spells of sickness absence

\begin{tabular}{|c|c|c|c|c|c|c|}
\hline \multirow[b]{3}{*}{ Diagnostic category (category code)* } & \multicolumn{6}{|c|}{ Mortality } \\
\hline & \multicolumn{2}{|c|}{ Cardiovascular (72 deaths) } & \multicolumn{2}{|c|}{ Neoplasms (144 deaths) } & \multicolumn{2}{|c|}{ Other (68 deaths) } \\
\hline & No of deaths & Hazard ratio $(95 \% \mathrm{Cl})$ & No of deaths & Hazard ratio $(95 \% \mathrm{Cl})$ & No of deaths & Hazard ratio $(95 \% \mathrm{Cl})$ \\
\hline No medically certified sickness absence & 36 & 1.00 & 80 & 1.00 & 41 & 1.00 \\
\hline $\begin{array}{l}\text { Any medically certified absence (all } \\
\text { diagnoses): }\end{array}$ & 36 & $1.96(1.20$ to 3.21$)$ & 64 & $1.73(1.22$ to 2.45$)$ & 27 & 1.39 (0.83 to 2.33$)$ \\
\hline Infectious and parasitic diseases (1) & 5 & $2.14(0.82$ to 5.60$)$ & 6 & 1.24 (0.53 to 2.88$)$ & 4 & $1.56(0.55$ to 4.46$)$ \\
\hline Circulatory (9-11) & 5 & $6.20(2.35$ to 16.3$)$ & 3 & $2.59(0.81$ to 8.29$)$ & 4 & $6.51(2.27$ to 18.7$)$ \\
\hline Diseases of respiratory system (12) & 15 & $2.23(1.18$ to 4.21$)$ & 21 & $1.52(0.92$ to 2.51$)$ & 10 & 1.40 (0.68 to 2.88$)$ \\
\hline $\begin{array}{l}\text { Diseases of musculoskeletal system and } \\
\text { connective tissue (17) }\end{array}$ & 3 & $0.98(0.30$ to 3.25$)$ & 5 & 0.93 (0.37 to 2.33$)$ & 4 & $1.40(0.49$ to 4.00$)$ \\
\hline Injury and poisoning (23) & 4 & $1.62(0.50$ to 4.67$)$ & 4 & 0.82 (0.30 to 2.28$)$ & 9 & 3.58 (1.68 to 7.66$)$ \\
\hline Surgical operations (37) & 8 & 2.85 (1.31 to 6.22$)$ & 12 & 1.94 (1.05 to 3.59$)$ & 7 & $2.19(0.97$ to 4.95$)$ \\
\hline Psychiatric $(40,41)$ & 3 & 1.24 (0.37 to 4.09$)$ & 12 & 2.49 (1.33 to 4.68$)$ & 4 & 1.61 (0.56 to 4.62$)$ \\
\hline Other $(2-8,13-16,20,35,38,39)$ & 8 & $2.16(0.98$ to 4.75$)$ & 12 & $1.63(0.87$ to 3.02$)$ & 7 & $1.83(0.80$ to 4.17$)$ \\
\hline Diagnosis not codeable or missing & 4 & $1.64(0.57$ to 4.69$)$ & 14 & 2.77 (1.54 to 4.97$)$ & 4 & $1.46(0.51$ to 4.16$)$ \\
\hline
\end{tabular}

cardiovascular mortality than cancer mortality, although not significantly so. However, absences with a musculoskeletal diagnosis were not associated with either cardiovascular or cancer mortality. None of the interactions between sex and diagnosis-specific absence were significant either for cancer mortality or cardiovascular mortality. Medically certified absences were associated with mortality from suicide (ICD-9 code E95, ICD-10 codes X60-X84) or external cause of undetermined intent (ICD-9 E98, ICD-10 Y10-Y34) (six deaths, hazard ratio $6.9,1.2$ to 39.1 ).

Adjustment for smoking, alcohol consumption, body mass index, and hypertension did not remove the associations of medically certified sickness absence with cause-specific mortality (table 4). As might be expected, the association between absences with a circulatory diagnosis and cardiovascular mortality was somewhat reduced after adjustment for these risk factors, but it remained significant (hazard ratio 3.4). However, adjustment for these risk factors only slightly attenuated the increased risk of cardiovascular mortality associated with absences for other diagnoses. Absences for both psychiatric and non-psychiatric causes maintained their associations with cancer mortality after adjustment for risk factors.

Exclusion of participants who died in the first three years after assessment of sickness absence had little effect on hazard ratios for cause-specific mortality (table 4). Similarly, when deaths in the first five years were excluded, there was still a twofold increase in cancer mortality among those having absences for psychiatric reasons.

\section{DISCUSSION}

This study shows that knowing the diagnosis for medically certified sickness absence from work significantly improves the prediction of mortality. Employees taking one or more medically certified spells of absence ( $>7$ days) for the common diagnostic categories had increased mortality compared with colleagues taking no medically certified absence. The only exception was musculoskeletal absence, which was not associated with increased mortality. Unexpectedly, employees who had one or more absence for psychiatric reasons had a considerable 2.5 -fold greater cancer mortality.

\section{Comparison with other studies}

Previous studies have shown that medically certified absence rates predict all cause mortality, ${ }^{38}$ but to our knowledge only one study, of very long term sickness absence $\left(>8\right.$ weeks), ${ }^{9}$ has linked diagnosis-specific sickness absence with mortality. While we found associations with mortality for most diagnosis-specific absences, this association was particularly strong for absences with a cardiovascular diagnosis and for absences with a psychiatric diagnosis, which is one of the most common diagnoses. We found no association between rates of absence for a musculoskeletal reason and mortality - which is as expected as musculoskeletal disorders rarely cause death. The increased risk of mortality among the few people who had absences because of cancer is in line with findings from the above mentioned study of long term sickness absence in Norway. ${ }^{9}$

Few studies on sickness absence have examined cause-specific mortality. We found that sickness absence predicts both cardiovascular and cancer mortality, the two leading causes of death in Western societies, replicating findings for Finnish municipal employees. ${ }^{8}$ In that Finnish study the association between sickness absence and cancer was stronger for cancers not attributable to smoking. In our study most of the cancer deaths $(70 \%)$ were not considered to be smoking related, ${ }^{7}$ and adjustment for smoking, alcohol consumption, and body mass index made little difference to our results, suggesting that the association between sickness absence and cancer mortality is not due to these risk factors. 
Table 4 | Hazard ratios for cause-specific mortality among civil service employees by specific diagnoses for medicallycertified spells of sickness absence

\begin{tabular}{|c|c|c|c|}
\hline \multirow[b]{2}{*}{ Diagnostic category* } & \multicolumn{3}{|c|}{ Hazard ratio $(95 \% \mathrm{Cl})$} \\
\hline & $\begin{array}{l}\text { Adjusted for age, sex, } \\
\text { and grade (model A) }\end{array}$ & $\begin{array}{l}\text { Model } A+\text { adjusted for smoking, alcohol, } \\
\text { body mass index, and hypertension }\end{array}$ & $\begin{array}{l}\text { Model A with three year } \\
\text { washout period } \dagger\end{array}$ \\
\hline Cardiovascular mortality & \multicolumn{2}{|c|}{ (68 deaths/6255 participants) } & $\begin{array}{l}\text { (59 deaths/ } \\
6218 \text { participants) }\end{array}$ \\
\hline No medically certified sickness absence & 1.00 & 1.00 & 1.00 \\
\hline Any medically certified absence (all diagnoses): & 2.24 (1.35 to 3.71$)$ & 1.97 (1.18 to 3.30$)$ & 2.18 (1.28 to 3.36$)$ \\
\hline Circulatory & $5.93(2.04$ to 17.3$)$ & $3.42(1.13$ to 10.4$)$ & $7.71(2.62$ to 22.7$)$ \\
\hline Other diagnoses & 2.08 (1.24 to 3.49$)$ & 1.88 (1.11 to 3.19) & $1.97(1.12$ to 3.46$)$ \\
\hline Cancer mortality & \multicolumn{2}{|c|}{ (134 deaths/6255 participants) } & $\begin{array}{c}\text { (118 deaths/ } \\
6218 \text { participants) }\end{array}$ \\
\hline No medically certified sickness absence & 1.00 & 1.00 & 1.00 \\
\hline Any medically certified absence (all diagnoses): & 1.84 (1.28 to 2.65$)$ & 1.75 (1.21 to 2.52$)$ & 1.83 (1.24 to 2.70$)$ \\
\hline Psychiatric & 2.64 (1.37 to 5.08$)$ & $2.44(1.26$ to 4.70$)$ & 2.27 (1.07 to 4.82$)$ \\
\hline Other diagnoses & 1.74 (1.19 to 2.54$)$ & 1.65 (1.13 to 2.43$)$ & 1.77 (1.18 to 2.65$)$ \\
\hline
\end{tabular}

*Modified RCGP morbidity coding system (see text for details).

tWashout period=exclusion of participants who died in the first three years after assessment of sickness absence.

Why is sickness absence with a psychiatric diagnosis related to all cause and cancer mortality? This was not due to confounding by health behaviours because adjustment for smoking and alcohol consumption hardly altered our results. Several studies have found that depression and negative affective dispositions, such as neuroticism and dispositional anxiety, are risk factors for all cause mortality and cardiovascular mortality. ${ }^{10-13}$ The large population based Norwegian HUNT study showed that depression predicted subsequent cancer mortality as well as cardiovascular mortality. ${ }^{14}$ One possible explanation for the association is that depression may interfere with help seeking behaviour, ${ }^{14}$ as survival is often related to early detection of cancer. A further possible explanation is that psychiatric disorders affect cancer prognosis rather than aetiology. ${ }^{15}$ Depression may also impair adherence to cancer treatment, although recent reviews on breast cancer found no conclusive evidence that psychological factors influence survival or relapses. ${ }^{1617}$ It is also possible that these psychiatric diagnoses are early symptoms of undiagnosed cancer. For example, the subcategory "neurosis ill-defined" included tiredness and stress, which could be early symptoms of undiagnosed cancer or other physical illness. However, excluding deaths in the first three years did not alter our results, and we still found a twofold increase in cancer mortality after we excluded deaths in the first five years.

\section{Strengths and limitations}

The strengths of our study were the large sample covering a wide range of employment grades from low to high incomes, the long follow-up for mortality, the use of reliable mortality registers, and the high validity of sickness absence data that included information on diagnosis.

Some limitations are noteworthy. Firstly, some data on sickness absence diagnosis were missing in the early years of the Whitehall II study. This is unlikely to have led to biased results, as associations of overall sickness absence with mortality from all causes, cancer, and cardiovascular disorders were similar in the total sample and in the sample analysed in this study.

Secondly, numbers of deaths were small in some of the diagnostic categories for sickness absence, and our results therefore need replication. There was insufficient power for detailed analyses of, for example, duration of sickness absence spells or less common diagnoses for absence. Our study also had low power to detect sex differences or to examine whether the association of sickness absence with mortality differed according to employment grade. Previous findings from this cohort showed that there are sex differences in rates of medically certified absences after taking account of age and employment grade: women had higher rates for absences with respiratory, psychiatric, or surgery diagnoses; similar rates for musculoskeletal and injury; and a non-significantly lower rate for cardiovascular diagnoses. ${ }^{6}$

Thirdly, the recorded diagnosis for a sickness absence may not cover all of the actual causes as sickness absence is a complex multifactorial behaviour and there may not be a simple relation between illness and taking of sick leave. ${ }^{18}$ Only one diagnosis is recorded for each sick leave, and thus coexisting diseases are not covered. Moreover, the stage and severity of disease when affected individuals decide to take sick leave may vary between diagnoses, and this might account for some of the differences in excess mortality between sickness absence diagnoses.

\section{Conclusions and policy implications}

Sickness absence is a serious economic problem with large costs for health care and many lost working hours for businesses. ${ }^{218} \mathrm{We}$ found that the almost 30\% of participants who had a sickness absence spell of more than seven days over a three year period had a $66 \%$ increased risk of premature death. We do not consider 


\section{WHAT IS ALREADY KNOWN ON THIS TOPIC}

Rates of medically certified sickness absence from work are associated with increased all cause mortality

It is not known whether this association is restricted to specific diagnoses for sickness absence

\section{WHAT THIS STUDY ADDS}

All the common diagnostic categories for sickness absence predicted overall mortality, with the exception of musculoskeletal diagnoses

Sickness absence with a psychiatric diagnosis, one of the commonest diagnoses for medically certified absence, was a strong predictor of cancer related mortality

Routinely collected data on sickness absences that include the diagnosis may help to identify groups at increased risk of fatal disease and allow targeted early interventions

that the taking of sick leave itself is a risk behaviour. Instead, it may be a marker of circumstances and health problems that increase mortality. We believe that better monitoring of diagnosis-specific absences at population levels may be useful and contribute to policy making-not only as a measure of costs but also as data to identify groups at increased health risk and in need of targeted intervention.

We thank all participating civil service departments and their welfare, personnel, and establishment officers; the Occupational Health and Safety Agency; the Council of Civil Service Unions; all participating civil servants in the Whitehall II study; all members of the Whitehall II study team. Contributors: All authors jointly designed the hypothesis, analysed and interpreted the data, and wrote the paper. JH is guarantor for the paper. Funding: The Whitehall II study has been supported by grants from the Medical Research Council; British Heart Foundation; Health and Safety Executive; Department of Health; National Heart Lung and Blood Institute (HL36310), US, NIH: National Institute on Aging (AG13196), US, NIH; Agency for Health Care Policy Research (HS06516); and the John D and Catherine T MacArthur Foundation Research Networks on Successful Midlife Development and Socio-economic Status and Health. JEF is supported by the MRC (grant No G8802774), KA is supported by the Swedish Council for Working life and Social Research, HW is supported by a programme grant from the Swedish Council for Working Life and Social Research (FAS grant No 2004-2021), and MK and JV are supported by the Academy of Finland (Projects No 117614, 124322, and 124271).

Competing interests: None declared.

Ethical approval: Ethical approval for the Whitehall II study was obtained from the University College London Medical School committee on the ethics of human research.
1 Marmot M, Feeney A, Shipley M, North F, Syme SL. Sickness absence as a measure of health status and functioning: from the UKWhitehall II study. J Epidemiol Community Health 1995;49:124-30.

2 Alexanderson K, Norlund A, (Eds.). Sickness absence-causes, consequences, and physicians' sickness certification practices: a systematic review. Scand I Public Health 2004;32(Suppl 63):1-263.

3 Kivimäki M, Head J, Ferrie JE, Shipley MJ, Vahtera J, Marmot MG Sickness absence as a global measure of health: evidence from mortality in the Whitehall II prospective cohort study. BMJ 2003;327:364.

4 Marmot MG, Davey Smith G, Stansfeld S, Patel C, North F, Head J, et al. Health inequalities among British civil servants: the Whitehall II study. Lancet 1991;337:1387-93.

5 Stansfeld SA, Feeney A, Head J, Canner R, North F, Marmot M. Sickness absence for psychiatric illness: the Whitehall II Study. Soc Sci Med 1995;40:189-97.

6 Feeney A, North F, Head J, Canner R, Marmot M. Socioeconomic and sex differentials in reason for sickness absence from the Whitehall II study. Occup Environ Med 1998;55:91-8.

7 Davey Smith G, Shipley M, Leon DA. Height and mortality from cancer among men: prospective observational study. $B M J$ 1998;317:1351-2.

8 Vahtera J, Pentti J, Kivimäki M. Sickness absence as a predictor of mortality among male and female employees. J Epidemiol Community Health 2004; 58:321-6.

9 Gjesdal S, Ringdal PR, Haug K, Maeland JG, Vollset SE, Alexanderson K. Mortality after long-term sickness absence: prospective cohort study. Eur J Public Health 2008. [Epub ahead of print]

10 Kubzansky LD, Kawachi I, Spiro A 3rd, Weiss ST, Vokonas PS, Sparrow D. Is worrying bad foryour heart? A prospective study of worry and coronary heart disease in the Normative Aging Study. Circulation 1997;95:818-24.

11 Eaker ED, Pinsky J, Castelli WP. Myocardial infarction and coronary death among women: psychosocial predictors from a 20-year followup of women in the Framingham Study. Am J Epidemiol 1992;135:854-64.

12 Goodwin RD, Cox BJ, Clara I. Neuroticism and physical disorders among adults in the community: results from the National Comorbidity Survey. J Behav Med 2006;29:229-38.

13 Suls J, Bunde J. Anger, anxiety, and depression as risk factors for cardiovascular disease: the problems and implications of overlapping affective dispositions. Psychol Bull 2005;131:260-300.

14 Mykletun A, Bjerkeset O, Dewey M, Prince M, Overland S, Stewart R. Anxiety, depression, and cause-specific mortality: the HUNT study. Psychosom Med 2007;69:323-31.

15 Speigel D, Giese-Davis J. Depression and cancer: mechanisms and disease progression. Biol Psychiatry 2003;54:269-82.

16 Nielson NR, Grøbaek M. Stress and breast cancer: a systematic update on the current knowledge. Nat Clin Pract Oncol 2006;3:612-20.

17 Falagas ME, Zarkadoulia EA, loannidou EN, Peppas G, Christodoulou C, Rafailidis PI. The effect of psychosocial factors on breast cancer outcome: a systematic review. Breast Cancer Res 2007;9:R44.

18 Henderson M, Glozier N, Elliot KH. Long term sickness absence. BMJ 2005;330:802-3.

Accepted: 15 July 2008 